\title{
HANDLEBODIES AND $p$-CONVEXITY
}

\author{
JI-PING SHA
}

The aim of this paper is to study the Riemannian geometry of manifolds with boundary. In a previous paper [4], the author proved the following theorem.

Let $M$ be a compact connected manifold with nonempty boundary. If $M$ admits a Riemannian metric with nonnegative sectional curvature and p-convex boundary, then $M$ has the homotopy type of a $C W$-complex of dimension $\leqslant p-1$.

Note. The author has recently learned that this theorem has also been proved independently by $\mathrm{H}$. $\mathrm{Wu}[5]$.

One of the main results of this paper is a converse of this theorem.

We begin by recalling the notion of $p$-convexity. Let $X$ be an $(n-1)$ dimensional (normally oriented) hypersurface in a Riemannian manifold $\Omega$ and let $\lambda_{1} \leqslant \lambda_{2} \leqslant \cdots \leqslant \lambda_{n-1}$ be its principal curvature functions. $X$ is called $p$-convex if $\lambda_{1}+\cdots+\lambda_{p}>0$ at each point of $X$. Note in particular that "1-convexity" is the usual notion of convexity; " $(n-1)$-convexity" means that $X$ has positive mean curvature. Also note that $p$-convexity implies $(p+1)$-convexity.

In [3], by a handle-attaching process, Lawson and Michelsohn showed the following: Suppose $X$ has positive mean curvature and let $X^{\prime}$ be a hypersurface obtained from $X$ by attaching an ambient $k$-handle to the positive side of $X$. If the codimension $(n-k)$ of the handle is $\geqslant 2$, then $X^{\prime}$ can be constructed also to have positive mean curvature. (That is to say that $X^{\prime}$ is ambiently isotopic to a hypersurface of positive mean curvature.)

Our central result is a generalization of this theorem to the $p$-convex case. Specifically we shall prove the following.

Theorem 1. Let $X$ be a (normally oriented) p-convex hypersurface in a Riemannian manifold $\Omega$, and let $X^{\prime}$ be a hypersurface obtained from $X$ by attaching a $k$-handle $D^{k}$ to the positive side of $X$. If $k \leqslant p-1$, then $X^{\prime}$ can be constructed also to be p-convex. 
Arguing as in [3] we get the following.

Corollary 2. Let $X$ be a compact manifold embedded as the boundary of a domain $D$ in a Riemannian manifold $\Omega$. Orient $X$ with respect to the inward pointing normal vector. If $D$ is diffeomorphic to a handlebody of dimension $\leqslant p-1$, then $X$ is ambiently isotopic through mutually disjoint embeddings to a $p$-convex hypersurface $X^{\prime}$ in $\Omega$. The new hypersurface $X^{\prime}$ bounds a domain $D^{\prime}$ which is diffeomorphic to $D$.

Applying this together with the fundamental results of Gromov in [1] we then obtain the following result which is a converse to the theorem in [4].

Theorem 3. Let $M$ be a compact connected manifold with nonempty boundary. If $M$ is a handlebody with handles only of dimension $\leqslant p-1$, then $M$ supports $a$ Riemannian metric with positive sectional curvature and p-convex boundary.

In fact, by the theorem of Gromov the sectional curvature of $M$ can be $\varepsilon$-pinched for any $\varepsilon>0$. If $M$ is parallelizable, then by immersion-submersion theory (cf. [2]) there exists an immersion $M \hookrightarrow S^{n}(1)$ where $n=\operatorname{dim} M$. By pulling back the constant curvature metric from $S^{n}(1)$ and proceeding as in Theorem 3, we have the following.

Theorem 4. Let $M$ be as in Theorem 3. If $M$ is parallelizable and is a handlebody with handles only of dimension $\leqslant p-1$, then $M$ supports a Riemannian metric with constant sectional curvature 1 and p-convex boundary.

The remainder of the paper is devoted to proving Theorem 1. Since our basic set-up closely follows Lawson and Michelsohn [3], our presentation will be brief. The basic picture is shown in Figure 1.

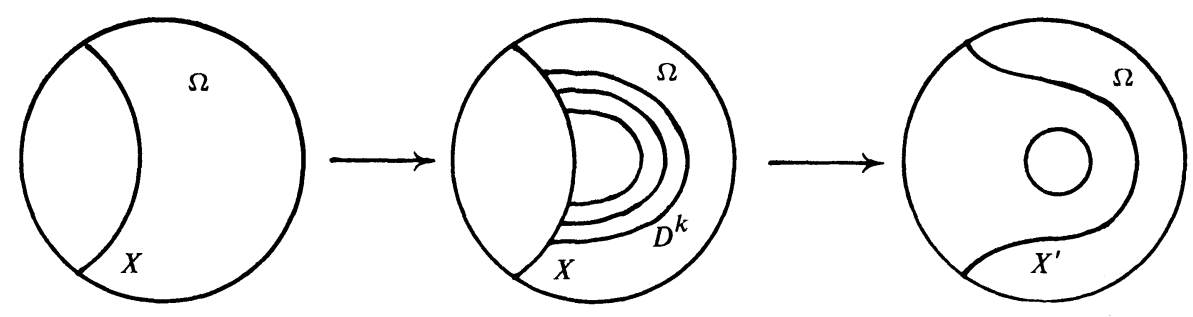

FIGURE 1

\section{The basic set-up}

Assume $\Omega$ is connected. Let $X$ be as in Theorem 1. Positive mean curvature (implied by $p$-convexity) implies a well-defined normal direction to $X$; i.e., we have an embedding of $X \times(-1,1)$ in $\Omega$ with the image of $X \times 0$ identified to $X$. Let $X^{+}$be the union of components of $\Omega \backslash X$ containing $X \times(0,1)$, and $X^{-}$be the union of components of $\Omega \backslash X$ containing $X \times(-1,0)$. 
Let $D^{k}$ be a $k$-dimensional disk orthogonally attached to $X$ in $X^{+}$. Set, for $x \in \Omega$,

$$
s(x) \equiv \operatorname{dist}(x, X), \quad r(x) \equiv \operatorname{dist}\left(x, D^{k}\right) .
$$

Then there exists a neighborhood $\Omega_{1}$ of $X$ in $\Omega$ such that $s$ is smooth in $\Omega_{1}^{\prime} \equiv \Omega_{1} \backslash X^{-}$and $\|\nabla s\| \equiv 1$. Similarly, there exists a neighborhood $\Omega_{2}$ of $D^{k}$ such that $r$ is smooth in $\Omega_{2}^{\prime} \equiv \Omega_{2} \backslash\left(X^{-} \cup D^{k}\right)$ and $\|\nabla r\| \equiv 1$. Then $r^{-1}\left(r_{0}\right) \cap$ $\Omega_{2}^{\prime}$ is a hypersurface in $\Omega_{2}^{\prime}$ for any sufficiently small $r_{0}>0$.

Hence, the map

$$
(r, s): \Omega_{1}^{\prime} \cap \Omega_{2}^{\prime} \rightarrow \mathbf{R}^{2}
$$

is a smooth submersion. Our idea is to construct a regular curve $\gamma$ which is essentially the graph of some function $s=f(r)$ in $\mathbf{R}^{2}$, so that the hypersurface $S_{\gamma} \equiv(r, s)^{-1}(\gamma)$ joins $r^{-1}\left(\varepsilon_{0}\right)$ to $X$ smoothly for some $\varepsilon_{0}>0$, and the whole new hypersurface obtained will still be $p$-convex.

Recall that the second fundamental form of the level hypersurface of a function is closely related to its Hessian form. We summarize this fact in the following.

Lemma 1. Let $u$ be a smooth function on a domain of $\Omega$. Then at every point the 2-form $\nabla^{2} u$ defined by

$$
\nabla^{2} u(\cdot, \cdot)=\operatorname{Hess}_{u}(\cdot, \cdot)=\langle\nabla \cdot(\nabla u), \cdot\rangle
$$

is symmetric. Furthermore, if $\|\nabla u\| \equiv 1$, then $\nabla u$ lies in the null space of $\nabla^{2} u$, and when restricted to $\nabla u^{\perp}, \nabla^{2} u$ is the second fundamental form of the level hypersurface of $u$ with respect to $-\nabla u$.

Proof. See [3]. q.e.d.

Suppose $u$ is a function as in Lemma 1 . Let

$$
\lambda_{1} \leqslant \lambda_{2} \leqslant \cdots \leqslant \lambda_{n}
$$

be the eigenvalues of $\nabla^{2} u$. We denote by $\sigma_{u}(m)$ the sum $\lambda_{1}+\cdots+\lambda_{m}$ for $m=1, \cdots, n$.

Remark. Note that by Lemma $1, \nabla u$ is an eigenvector of $\nabla^{2} u$, the corresponding eigenvalue is 0 . The other $(n-1)$ eigenvalues are the principal curvatures of the level hypersurface of $u$. We then clearly have that the level hypersurface is $p$-convex if and only if $\sigma_{u}(p+1)$ is positive.

Lemma 2. (i) We can choose $\Omega_{1}$ such that there exists a constant $\delta>0$ for which $\sigma_{s}(p+1)>\delta$ in $\Omega_{1}$. (Here $\delta$ could be replaced by a smooth positive function.)

(ii) We can choose $\Omega_{2}$ such that $\sigma_{r}(p+1)>c / r$ in $\Omega_{2} \backslash\left(X^{-} \cup D^{k}\right)$, where $c>0$ is a constant.

Proof. (i) is from the $p$-convexity of $X$.

(ii) is by a calculation in Fermi coordinates and the fact that $k \leqslant p-1$ as follows. 
Choose locally smooth orthonormal vector fields $e_{1}, \cdots, e_{n}$ along $D^{k}$ such that $e_{1}, \cdots, e_{k}$ are tangent to $D^{k}$ and that $e_{k+1}, \cdots, e_{n}$ are normal to $D^{k}$. Then for $\xi \in D^{k},\left(x_{1}, \cdots, x_{n-k}\right) \in \mathbf{R}^{n-k}$ with $x_{1}^{2}+\cdots+x_{n-k}^{2}$ small, the map

$$
\left(\xi,\left(x_{1}, \cdots, x_{n-k}\right)\right) \mapsto \exp _{\xi}\left(x_{1} e_{k+1}+\cdots+x_{n-k} e_{n}\right)
$$

gives a local coordinate in some open set $W \subset \Omega_{2}$. Extend $e_{1}, \cdots, e_{n}$ to smooth vector fields $\tilde{e}_{1}, \cdots, \tilde{e}_{n}$ on $W$, where each $\tilde{e}_{i}$ is obtained by parallel translation of $e_{i}$ along the geodesic

$$
\alpha(t)=\exp _{\xi}\left[t\left(x_{1} e_{k+1}+\cdots+x_{n-k} e_{n}\right)\right], \quad 0 \leqslant t \leqslant 1 .
$$

On $W$, it is clear that

$$
r\left(\xi,\left(x_{1}, \cdots, x_{n-k}\right)\right)=\sqrt{x_{1}^{2}+\cdots+x_{n-k}^{2}}
$$

and that

$$
\nabla r=\frac{1}{r}\left(x_{1} \tilde{e}_{k+1}+\cdots+x_{n-k} \tilde{e}_{n}\right) .
$$

If the metric were Euclidean, i.e., if all the $\tilde{e}_{i}$ 's were parallel, we would obviously have

$$
\sigma_{r}(p+1)=(p-k) / r .
$$

In general, let $V_{1}, \cdots, V_{p+1}$ be arbitrary $(p+1)$ orthonormal tangent vectors at some point in $W$. We have that

$$
\begin{aligned}
\sum_{i=1}^{p+1} \nabla^{2} r\left(V_{i}, V_{i}\right)= & \sum_{i=1}^{p+1} \tilde{\nabla}^{2} r\left(V_{i}, V_{i}\right) \\
& +\sum_{i=1}^{p+1}\left(\frac{x_{1}}{r}\left\langle\nabla_{V_{i}} \tilde{e}_{k+1}, V_{i}\right\rangle+\cdots+\frac{x_{n-k}}{r}\left\langle\nabla_{V_{i}} \tilde{e}_{n}, V_{i}\right\rangle\right),
\end{aligned}
$$

where $\tilde{\nabla}^{2} r$ denotes the Hessian of $r$ under the Euclidean metric. Then the first sum in $(*)$ is $\geqslant(p-k) / r$. The second sum in $(*)$ can clearly be bounded by some fixed constant which is independent of $r$. Therefore by choosing $\Omega_{2}$ properly and noting that $p-k \geqslant 1$, there exists a constant $c>0$ such that

$$
\sigma_{r}(p+1)>c / r
$$

in $\Omega_{2} \backslash\left(X^{-} \cup D^{k}\right)$.

\section{The bending function}

Let $\delta, \varepsilon_{1}, \varepsilon_{2}$, and $c_{0}$ be fixed positive constants. Our aim in this section is to construct a smooth function $f$ which is defined on $r>\varepsilon_{0}$ for some $0<\varepsilon_{0}<\varepsilon_{1}$ such that

$$
\begin{array}{ll}
f(r)=0 & \text { for } r \geqslant \varepsilon_{1} \\
f^{\prime}(r) \leqslant 0 & \text { for } r>\varepsilon_{0} \\
f(r) \rightarrow \varepsilon_{3}<\varepsilon_{2} & \text { as } r \rightarrow \varepsilon_{0}^{+} .
\end{array}
$$




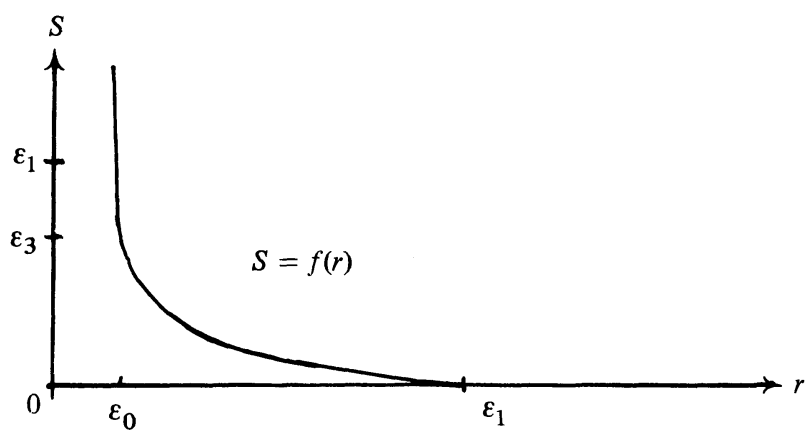

FIGURE 2
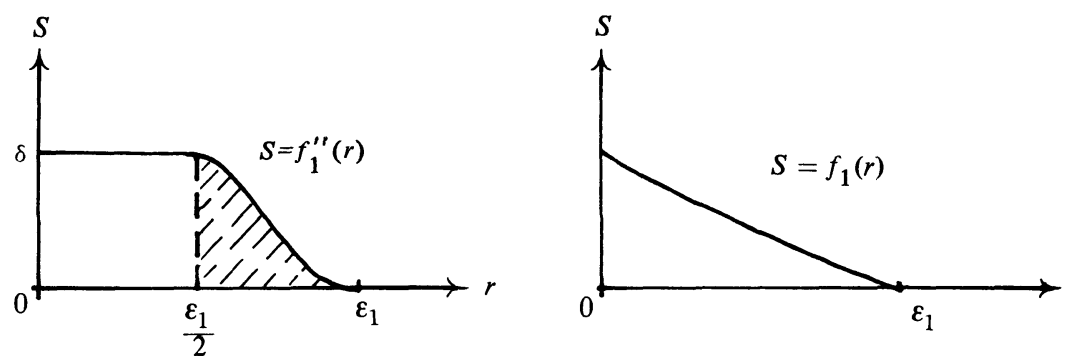

Figure 3

All the derivatives of $f \rightarrow \infty$ in absolute value as $r \rightarrow \varepsilon_{0}^{+}$(see Figure 2).

Furthermore, $f$ satisfies either of the following conditions for $r>\varepsilon_{0}$ :

$$
\delta-f^{\prime \prime}(r)-\frac{c_{0} f^{\prime}(r)}{r}>0 \text { or } \delta-\frac{f^{\prime \prime}(r)}{f^{\prime}(r)^{2}}-\frac{c_{0} f^{\prime}(r)}{r}>0 .
$$

We begin by choosing $f_{1}^{\prime \prime}$ properly to get a smooth function $f_{1}$ such that

$$
\begin{array}{cc}
f_{1}(r)=0 & \text { for } r \geqslant \varepsilon_{1} ; \\
f_{1}^{\prime}(r) \leqslant 0 & \text { for all } r ; \\
0<f_{1}^{\prime \prime}(r)=\text { constant }<\delta & \text { for } r<\varepsilon_{1} / 2 ; \\
\exp \left[\frac{1}{2 c_{0} f_{1}^{\prime}\left(\varepsilon_{1} / 2\right)^{2}}\right]>1 ; & \frac{c_{0}\left[-f_{1}^{\prime}\left(\varepsilon_{1} / 2\right)\right]^{3}}{f_{1}^{\prime \prime}(0)}<\frac{\varepsilon_{1}}{2} ; \\
f_{1}(0)+\frac{c_{0}\left[-f_{1}^{\prime}\left(\varepsilon_{1} / 2\right)\right]^{3}}{f_{1}^{\prime \prime}(0)} \cdot \frac{1}{l} \int_{1}^{l} \frac{d t}{\sqrt{2 c_{0} \ln t}}<\varepsilon_{2} \quad \text { for all } l>1 .
\end{array}
$$


All the requirements can be satisfied by choosing $f_{1}^{\prime \prime}(0)$ small and then by choosing the area of the shaded part in Figure 3 small and also by noting that

$$
\frac{1}{l} \int_{1}^{l} \frac{d t}{\sqrt{2 c_{0} \ln t}} \rightarrow 0 \text { as } l \rightarrow \infty ;
$$

therefore, in particular, it is bounded for $l>1$.

Now set

$$
a=\exp \left[\frac{1}{2 c_{0} f_{1}^{\prime}\left(\varepsilon_{1} / 2\right)^{2}}\right], \quad \varepsilon_{0}=\frac{c_{0}\left[-f_{1}^{\prime}\left(\varepsilon_{1} / 2\right)\right]^{3}}{a f_{1}^{\prime \prime}(0)} .
$$

Then $a>1$ and $a \varepsilon_{0}<\varepsilon_{1} / 2$ by the construction of $f_{1}$.

Define for $r>\varepsilon_{0}$

$$
f_{2}(r)=\int_{r}^{a \varepsilon_{0}} \frac{d t}{\sqrt{2 c_{0} \ln \left(t / \varepsilon_{0}\right)}}
$$

We have

$$
f_{2}^{\prime}(r)=-\frac{1}{\sqrt{2 c_{0} \ln \left(r / \varepsilon_{0}\right)}}, \quad f_{2}^{\prime \prime}(r)=\frac{1}{2 \sqrt{2 c_{0}}\left(\ln \left(r / \varepsilon_{0}\right)\right)^{3 / 2} r} .
$$

Hence

Finally, let

$$
\frac{f_{2}^{\prime \prime}(r)}{f_{2}^{\prime}(r)^{2}}+\frac{c_{0} f_{2}^{\prime}(r)}{r}=0
$$

$$
f_{3}(r)= \begin{cases}f_{1}\left(\varepsilon_{1} / 2\right)+f_{2}(r) & \text { for } \varepsilon_{0}<r \leqslant a \varepsilon_{0}, \\ f_{1}\left(r-a \varepsilon_{0}+\varepsilon_{1} / 2\right) & \text { for } r \geqslant a \varepsilon_{0} .\end{cases}
$$

Then it is easy to verify that $f_{3}$ is $C^{2}$ and satisfies all the conditions required for $f$. In fact when $r \geqslant a \varepsilon_{0}$

$$
\delta-f_{3}^{\prime \prime}(r)-\frac{c_{0} f_{3}^{\prime}(r)}{r}>0
$$

by the construction of $f_{1}$ and when $\varepsilon_{0}<r \leqslant a \varepsilon_{0}$

$$
\delta-\frac{f_{3}^{\prime \prime}(r)}{f_{3}^{\prime}(r)^{2}}-\frac{c_{0} f_{3}^{\prime}(r)}{r}=\delta-\frac{f_{2}^{\prime \prime}(r)}{f_{2}^{\prime \prime}(r)^{2}}-\frac{c_{0} f_{2}^{\prime}(r)}{r}=\delta>0 .
$$

The required $f$ is then gotten by a smoothing of $f_{3}$.

\section{The construction of $X^{\prime}$}

Let $D_{\varepsilon}=\{x \in \Omega: \quad r(x)<\varepsilon\}$ and $X_{\varepsilon}=\{x \in \Omega: s(x)<\varepsilon\}$ be tubular neighborhoods of $D^{k}$ and $X$ respectively.

There exist $\varepsilon_{1}, \varepsilon_{2}>0$ such that $D_{2 \varepsilon_{1}} \subset \Omega_{2}, X_{2 \varepsilon_{2}} \subset \Omega_{1}$ and such that $|\langle\nabla r, \nabla s\rangle|<1$ in $U=\left\{x \in D_{2 \varepsilon_{1}} \cap X_{2 \varepsilon_{2}} \cap X^{+}: r(x)>0\right\}$. 
Let $\gamma$ be the curve $s=f(r)$ as in Figure 2. The hypersurface $S_{\gamma}=(r, s)^{-1}(\gamma)$ smoothly joining $X \backslash(X \cap U)$ to $\partial D_{\varepsilon_{0}} \backslash\left(\partial D_{\varepsilon_{0}} \cap U\right)$ produces a new hypersurface which will be our hypersurface $X^{\prime}$ obtained from $X$ by attaching the handle $D^{k}$ (see Figure 4).

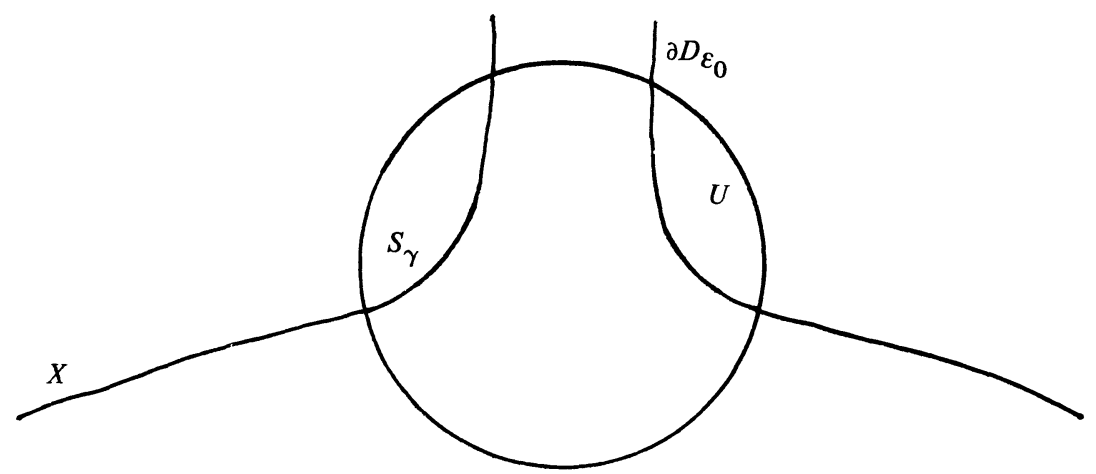

Figure 4

We claim that $X^{\prime}$ is $p$-convex. It only needs to be verified at the part of $S_{\gamma}$ where $r>\varepsilon_{0}$. For this part, $S_{\gamma}$ is the level set of the smooth function $F(x)=s(x)-f(r(x))$.

We have

$$
\begin{aligned}
& \nabla F=\nabla s-f^{\prime}(r) \nabla r, \\
& \nabla^{2} F=\nabla^{2} s-f^{\prime}(r) \nabla^{2} r-f^{\prime \prime}(r)(\nabla r)^{2} .
\end{aligned}
$$

Let $e_{n}=\nabla F /\|\nabla F\|$. The second fundamental form of $S_{\gamma}$ is given by

$$
B_{F}(\cdot, \cdot)=\left\langle\nabla \cdot e_{n}, \cdot\right\rangle=\frac{\nabla^{2} F}{\|\nabla F\|}-\frac{\nabla\left(\|\nabla F\|^{2}\right) \nabla F}{2\|\nabla F\|^{3}}
$$

Clearly $B_{F}\left(e_{n}, e_{n}\right)=0$ and

$$
\begin{aligned}
& \nabla_{e_{n}}\left(\|\nabla F\|^{2}\right) \nabla_{e_{n}} F=\nabla_{e_{n}}\left(\|\nabla F\|^{2}\right)\left\langle e_{n}, \nabla F\right\rangle \\
& =\frac{\nabla_{\nabla F}}{\|\nabla F\|}\langle\nabla F, \nabla F\rangle \cdot\left\langle\frac{\nabla F}{\|\nabla F\|}, \nabla F\right\rangle \\
& =2\left\langle\nabla_{\nabla F} \nabla F, \nabla F\right\rangle=2 \nabla^{2} F(\nabla F, \nabla F) \\
& =2\left[\nabla^{2} s(\nabla F, \nabla F)-f^{\prime}(r) \nabla^{2} r(\nabla F, \nabla F)\right]-2\|\nabla F\|^{2} f^{\prime \prime}(r)\left(\nabla_{e_{n}} r\right)^{2} \\
& =2\left[f^{\prime}(r)^{2} \nabla^{2} s(\nabla r, \nabla r)-f^{\prime}(r) \nabla^{2} r(\nabla s, \nabla s)\right]-2\|\nabla F\|^{2} f^{\prime \prime}(r)\left(\nabla_{e_{n}} r\right)^{2},
\end{aligned}
$$


where the last equality is obtained by recalling that $\nabla s$ is in the null space of $\nabla^{2} s$ and that $\nabla r$ is in the null space of $\nabla^{2} r$.

Then

$$
\begin{aligned}
\frac{\nabla_{e_{n}}\left(\|\nabla F\|^{2}\right) \nabla_{e_{n}} F}{2\|\nabla F\|^{3}}= & -\frac{f^{\prime \prime}(r)}{\|\nabla F\|}\left(\nabla_{e_{n}} r\right)^{2} \\
& -\frac{1}{\|\nabla F\|^{3}}\left[f^{\prime}(r) \nabla^{2} r(\nabla s, \nabla s)-f^{\prime}(r)^{2} \nabla^{2} s(\nabla r, \nabla r)\right] .
\end{aligned}
$$

Now suppose that $e_{1}, \cdots, e_{p}$ are orthonormal vectors tangent to $s_{\gamma}$. Then $\nabla_{e_{i}} F=0$ for $i=1, \cdots, p$.

Therefore

$$
\begin{aligned}
& \sum_{i=1}^{p} B_{F}\left(e_{i}, e_{i}\right)=\sum_{i=1}^{p} B_{F}\left(e_{i}, e_{i}\right)+B_{F}\left(e_{n}, e_{n}\right) \\
& =\frac{1}{\|\nabla F\|} \sum_{i=1}^{p}\left[\nabla^{2} s\left(e_{i}, e_{i}\right)-f^{\prime}(r) \nabla^{2} r\left(e_{i}, e_{i}\right)-f^{\prime \prime}(r)\left(\nabla_{e_{i}} r\right)^{2}\right] \\
& +\frac{1}{\|\nabla F\|}\left[\nabla^{2} s\left(e_{n}, e_{n}\right)-f^{\prime}(r) \nabla^{2} r\left(e_{n}, e_{n}\right)-f^{\prime \prime}(r)\left(\nabla_{e_{n}} r\right)^{2}\right] \\
& +\frac{1}{\|\nabla F\|} f^{\prime \prime}(r)\left(\nabla_{e_{n}} r\right)^{2} \\
& +\frac{1}{\|\nabla F\|^{3}}\left[f^{\prime}(r) \nabla^{2} r(\nabla s, \nabla s)-f^{\prime}(r)^{2} \nabla^{2} s(\nabla r, \nabla r)\right] \\
& \geqslant \frac{1}{\|\nabla F\|}\left[\sigma_{s}(p+1)-f^{\prime}(r) \sigma_{r}(p+1)-f^{\prime \prime}(r) \sum_{i=1}^{p}\left(\nabla_{e_{i}} r\right)^{2}\right] \\
& +\frac{1}{\|\nabla F\|^{3}}\left[f^{\prime}(r) \nabla^{2} r(\nabla s, \nabla s)-f^{\prime}(r)^{2} \nabla^{2} s(\nabla r, \nabla r)\right] \\
& \geqslant \frac{1}{\|\nabla F\|}\left[\delta-f^{\prime}(r)\left(\frac{c}{r}-\frac{1}{\|\nabla F\|^{2}}\left|\frac{r \nabla^{2} r(\nabla s, \nabla s)}{r}\right|\right.\right. \\
& \left.\left.-\frac{1}{\|\nabla F\|^{2}}\left|f^{\prime}(r) \nabla^{2} s(\nabla r, \nabla r)\right|\right)-f^{\prime \prime}(r) \sum_{i=1}^{p}\left(\nabla_{e_{i}} r\right)^{2}\right] \text {. }
\end{aligned}
$$

Note that

$$
\lim _{r \rightarrow 0} r \nabla^{2} r(\nabla s, \nabla s)=0
$$


in $U$, and that

$$
\nabla^{2} s(\nabla r, \nabla r), \quad \frac{f^{\prime}(r)}{\|\nabla F\|^{2}}=\frac{f^{\prime}(r)}{1+f^{\prime}(r)^{2}-2 f^{\prime}(r)\langle\nabla r, \nabla s\rangle}
$$

are bounded in $U$. It is then easy to see that we can choose $\varepsilon_{1}, \varepsilon_{2}, c_{0}$ so that

$$
\sum_{i=1}^{p} B_{F}\left(e_{i}, e_{i}\right) \geqslant \frac{1}{\|\nabla F\|}\left[\delta-\frac{c_{0} f^{\prime}(r)}{r}-f^{\prime \prime}(r)\right]
$$

or (note that $\nabla_{e_{i}} r=\nabla_{e_{i}} s / f^{\prime}(r)$ )

$$
\sum_{i=1}^{p} B_{F}\left(e_{i}, e_{i}\right) \geqslant \frac{1}{\|\nabla F\|}\left[\delta-\frac{c_{0} f^{\prime}(r)}{r}-\frac{f^{\prime \prime}(r)}{f^{\prime}(r)^{2}}\right] .
$$

Therefore by the construction of $f, s_{\gamma}$ is $p$-convex.

\section{References}

[1] M. Gromov, Stable mappings of foliations into manifolds, Izv. Akad. Nauk SSSR Ser. Mat. 33 (1969) 707-734; English transl., Math. USSR-Izv. 3 (1969) 671-694.

[2] M. Hirsch, Immersions of manifolds, Trans. Amer. Math. Soc. 93 (1959) 242-276.

[3] B. Lawson \& M-L. Michelsohn, Embedding and surrounding with positive mean curvature, Invent. Math. 77 (1984) 399-419.

[4] J. Sha, p-convex riemannian manifolds, Invent. Math. 83 (1986) 437-447.

[5] H. Wu, Manifolds of partially positive curvature, preprint.

State University of New York, Stony Brook 
